\title{
Experimental validation of elliptical fin-opening behavior
}

\author{
James M. Garner*, Paul Weinacht and Robert P. Kaste \\ U.S. Army Research Laboratory, Bldg 390 Aberdeen Proving Ground, MD, USA 21005
}

\begin{abstract}
An effort to improve the performance of ordnance has led to the consideration of the use of folding elliptical fins for projectile stabilization. A second order differential equation was used to model elliptical fin deployment history and accounts for: deployment with respect to the geometric properties of the fin, the variation in fin aerodynamics during deployment, the initial yaw effect on fin opening, and the variation in deployment speed based on changes in projectile spin. This model supports tests conducted at the Transonic Experimental Facility, Aberdeen Proving Ground examining the opening behavior of these uniquely shaped fins. The fins use the centrifugal force from the projectile spin to deploy. During the deployment, the fin aerodynamic forces vary with angle-of-attack changes to the free stream. Model results indicate that projectile spin dominates the initial opening rates and aerodynamics dominate near the fully open state. The model results are examined to explain the observed behaviors, and suggest improvements for later designs.
\end{abstract}

\section{Introduction}

Proposals to create a composite artillery projectile have arisen recently. A prime benefit of such a round would be a roughly $25 \%$ reduction in projectile weight. The weight reduction has significant benefits in terms of transportation and loading/handling of the projectiles. A proposed composite artillery projectile offered by the Army Research Laboratory employs fins as an aerodynamic stabilization method. Spin stabilization, as is the present standard for artillery projectiles, would require extensive engineering and specialized fabrication to withstand the torque loads applied during inbore spin-up. Additionally, the mass distribution for efficient spin stabilization in a lightweight projectile would be unfavorable for use with the current projectile payloads.

An alternative to conventional fins, either fixed, folding, or wrap around, is offered with the use of elliptical, deployable fins. These fins (dubbed Kayser fins in honor of their creator, Lyle Kayser) combined with their inherent boattail reduce the increase in drag associated

\footnotetext{
*Corresponding author: Tel.: +410 278 6557; Fax: +410 2782460; E-mail: garner@arl.army.mil.
}

with the addition of fins to a previously unfinned body. The typical Kayser four fin configuration is shown in Fig. 1. The fins are stowed in-bore and are designed to fully deploy to 135 degrees (2.36 radians) on muzzle exit. The fins weigh approximately 175 grams each and have an area of 84 square centimeters.

When stowed, they are folded against the boattail and this configuration allows more efficient use of the boattail volume. For example, the boattail volume may now be configured to house a rocket motor similar to that of the M549. Typically, stowed folding fins intrude into the body/boattail and clearly would not allow such an option. The deployment properties of Kayser fins have been the described previously [3]. These previous descriptions have relied on controlled static tests as the fin design has only recently been ballistically tested. The fin opening behavior is primarily governed by controllable factors such as fin mass properties, fin cant angles, and projectile spin. Less controllable factors such as yaw states at muzzle exit also affect the fin deployment. The effect of yaw is generally to inhibit the deployment of fins on the windward side and enhance deployment on the leeward side. This report offers insight on the effect of aerodynamic loads encountered at launch on fin opening via the use of the experimental 

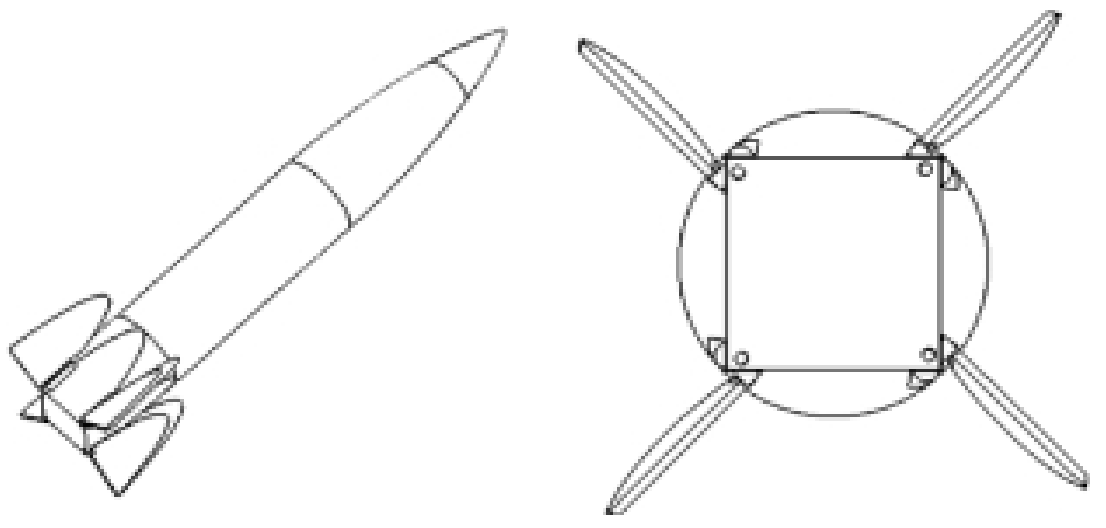

Fig. 1. Isometric and rear view of the $34 \mathrm{~kg}$ projectile.

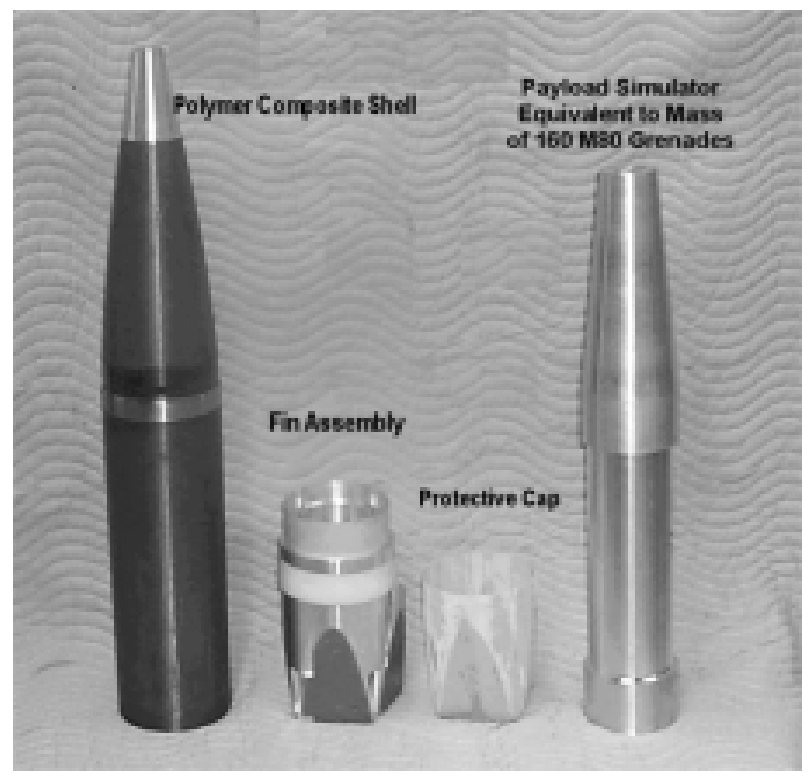

Fig. 2. Components of a $34 \mathrm{~kg}$ test projectile.

data coupled with the solution of equations given in previous analytical models.

\section{Design aspects}

Fin stabilized artillery projectiles with fixed fin designs would be very inefficient. They would require large sabot volumes and mandate large cannons. Deployable fins are clearly necessary for artillery shells if they are going to retain their same basic geometry and be fired from existing $155 \mathrm{~mm}$ cannons. For the Kayser fin configuration, the fin area and boattail design are integrated such that they provide an adequate fin area for stabilization as well as a boattail that reduces base drag in addition to being deployable. A 7 degree boattail angle was selected based on a previous Ballistic Research Laboratory Report indicating it to be near optimal from an aerodynamic drag standpoint [1]. This boattail in the aforementioned report was axisymmetric, but it offers confirmation to some extent that this same angle is suitable for the Kayser fin boattail. This angle provides sufficient fin area to stabilize the projectile. Another criterion to be met is the deployment performance of the fin once it exits the gun. Figure 2 shows the components of a projectile utilized in ballistic testing. This complemented the modeling efforts.

Knowing the range of motion for the fin is critical if it is expected to sweep through a 2.36 radians ( $135 \mathrm{de}$ grees) angle and lock into position. Fortunately, there 
is some latitude in the design parameters of the fin. The properties of the fin have some latitude in their design as stability requirements do not impose a unique design. The appropriate amount of over-design to mitigate random launch conditions and assure consistent fin deployment is not well determined. Random launch conditions such as muzzle exit yaw and pitch angles affect the aerodynamic force, $F_{\text {aero }}$ on the fins. Observations of this behavior are from actual firings, and are presented here.

In a previous effort, Kayser and Brown developed a simplified model of the fin opening event [3]. Their analysis resulted in the following equation of motion for the fin blade:

$$
\ddot{\theta}+\frac{0.8 b \omega^{2}}{a} \sin \theta=\frac{0.8 F_{\text {aero }}}{m a}
$$

with:

a Fin half-height $(a=0.049 \mathrm{~m})$

b Projectile axis to pivot point distance $(b=$ $0.072 \mathrm{~m})$

$\mathrm{m}$ Fin mass $(0.175 \mathrm{~kg})$

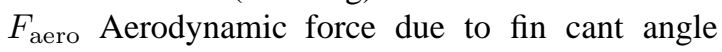
(tends to close the fully opened fin)

$\theta$ Angle between projectile body and fin face

$\omega$ Projectile rotational rate

This ordinary differential Eq. (1) describes the fin opening angle, $\theta$, in terms of the geometric, mass and aerodynamic properties of the fin illustrated in Fig. 3. The equation is a close analog to the equation of a pendulum Eq. (2) with the exception that the equation of motion for the fin opening includes a forcing function on the right hand side. The forcing function is produced by the applied (aerodynamic) forces acting on the fin blade:

$$
\ddot{\theta}+\frac{g}{l} \sin \theta=0 .
$$

The aerodynamic forces acting on the fin blade during the fin opening event can be complicated due to the three-dimensional flow field produced by the interaction of the fins and the projectile body during launch and flight. To make the problem tractable, Kayser and Brown made the following assumptions: (1) the aerodynamic force acting on the fin blade was proportional to the local cant angle of the fin relative to the free stream flow and (2) the aerodynamic force was related to the roll producing moment produced by the fin cant.

Kayser and Brown related the aerodynamic force in the following manner;

$$
F_{\text {aero }}=\frac{F_{\max }}{\delta_{f}} \delta_{r}
$$

where $\delta_{r}$ is the instantaneous effective cant angle of the fin blade and $F_{\max }$ is the aerodynamic lift force produced by the fin when it is fully deployed to the final cant angle $\delta_{f}$.

Kayser and Brown found that for their fin geometry, the effective fin cant angle could be approximated by a simple cosine function. In the current effort, a formal derivation of the fin cant angle in terms of two hinge angles, the projectile boattail angle, the free stream angle of attack and the fin opening angle is presented. From this analysis, it is found that the approximated form of the fin cant angle used by Kayser and Brown is not universally valid. Furthermore, by including two angles to describe the hinge line for the fin blade, additional control of the aerodynamic properties of the fin (including the fin cant angle when the fin is fully open) is possible. In addition, free stream angle of attack appears to significantly affect on the fin opening event.

The local fin cant angle is defined as the enclosed angle between the free stream velocity vector and the vector normal to the fin surface and is related as follows:

$$
|\vec{U}| \sin \delta_{r}=\vec{U} \cdot \vec{n}
$$

To compute the fin cant angle, both the fin normal vector $\vec{n}$ and the fin stream velocity vector $\vec{U}$ must be determined.

The fin normal vector is purely a function of the geometric properties of the fin. The current derivation assumes that the boattail and fins are cut symmetrically. The hinge line for the fin blade is described by two compound angles shown in Fig. 4. Note also that the first compound angle $\gamma_{1}$, for the hinge is not required to have the same angle as the projectile boattail. This allows for additional control of the fin aerodynamic to enhance the fin opening. The fin normal vector has the following form:

$$
\begin{aligned}
\vec{n}= & \tilde{i}\left(\cos \gamma_{1} \cos \gamma_{2}^{\prime} \cos \gamma_{4} \sin \gamma_{5}\right. \\
& +\cos \gamma_{1} \sin \gamma_{2}^{\prime} \cos (3 \pi / 4-\theta) \sin \gamma_{4} \sin \gamma_{5} \\
& +\sin \gamma_{1} \sin (3 \pi / 4-\theta) \sin \gamma_{4} \sin \gamma_{5} \\
& -\cos \gamma_{1} \sin \gamma_{2}^{\prime} \sin (3 \pi / 4-\theta) \cos \gamma_{5} \\
& \left.+\sin \gamma_{1} \cos (3 \pi / 4-\theta) \cos \gamma_{5}\right) \\
& +\tilde{j}\left(-\sin \gamma_{2}^{\prime} \cos \gamma_{4} \sin \gamma_{5}\right. \\
& +\cos \gamma_{2}^{\prime} \cos (3 \pi / 4-\theta) \sin \gamma_{4} \sin \gamma_{5} \\
& \left.-\cos \gamma_{2}^{\prime} \sin (3 \pi / 4-\theta) \sin \gamma_{5}\right) \\
& +\tilde{k}\left(\sin \gamma_{1} \cos \gamma_{2}^{\prime} \cos \gamma_{4} \sin \gamma_{5}\right.
\end{aligned}
$$




$$
\begin{aligned}
& -\sin \gamma_{1} \sin \gamma_{2}^{\prime} \cos (3 \pi / 4-\theta) \sin \gamma_{4} \sin \gamma_{5} \\
& +\cos \gamma_{1} \sin (3 \pi / 4-\theta) \sin \gamma_{4} \sin \gamma_{5} \\
& -\sin \gamma_{1} \sin \gamma_{2}^{\prime} \sin (3 \pi / 4-\theta) \cos \gamma_{5} \\
& \left.+\cos \gamma_{1} \cos (3 \pi / 4-\theta) \cos \gamma_{5}\right) \\
\gamma_{2}= & \tan ^{-1}\left(\tan \gamma_{2}^{\prime} / \cos \gamma_{1}\right) \\
\gamma_{4}= & \gamma_{2}^{\prime} \\
\gamma_{5}= & \gamma_{B T}-\gamma_{1}
\end{aligned}
$$

The freestream velocity vector has the following form,

$$
\begin{aligned}
\vec{U}= & U_{\infty}\{\tilde{i}(\cos \alpha)+\tilde{j}(\sin \alpha \sin \phi) \\
& +\tilde{k}(\sin \alpha \cos \phi)\}
\end{aligned}
$$

where $U_{\infty}$ is the magnitude of the freestream velocity, $\alpha$ is the angle of attack and $\phi$ is the orientation angle of the pitch plane (shown in Fig. 3).

This model does not consider the three dimensional nature of the flow field in the vicinity of the fins. A comprehensive modeling of this region using computation fluid dynamics is possible but entails significant complexity and effort. The current model assumes that the local angle-of-attack is represented by the free stream angle-of-attack and ignores three dimensional effects such as those produced by the wake of a body. For the small angles of attack observed (less than 5 degrees) these effects are assumed to be minimal because the flow over the projectile remains attached. Though simplified, the current model incorporates the effect of angle of attack experimentally observed and represents an improvement over existing models.

Figure 5 shows the fin cant angle as a function of the fin opening angle for the conceptual design of Kayser and Brown versus the design most recently fired, for zero pitch and yaw angles. Geometric parameters for each design are shown in Table 1. (Kayser and Brown provide limited details of the geometric configuration of their design, in particular, the hinge line. The details of this design used in the current analysis have been reconstructed from some of the results published originally by Kayser and Brown.) Also shown is the simple cosine approximation form of the fin cant angle proposed by Kayser and Brown. Clearly, the effective cant angle for the current design would be poorly approximated by the simple cosine function. The variation in free stream angle induced by the fin angular velocity as it opens is not included in Fig. 5 as its effect on angle-of-attack is minimal (less than a degree) and varies over the fin span. Also of note is the definition
Table 1

Hinge and boattail angles

\begin{tabular}{lcc}
\hline & Current Design & Kayser and Brown \\
\hline$\gamma_{1}$ (Degrees) & 5. & 6. \\
$\gamma_{2}$ (Degrees) & 2. & 4. \\
$\gamma_{B T}$ (Degrees) & 7. & 6. \\
\hline
\end{tabular}

of $\theta$ (the fin opening angle). $\theta=0$ degrees is the state where the fin is fully deployed, and conversely $\theta=135$ degrees is the state where the fin is closed against the body.

Figure 5 also shows that early in the opening event $(\theta \approx 135$ degrees), the fin cant angle is negative resulting in an aerodynamic force that tends to open the fin. At larger fin opening angles, the fin cant angle changes sign and the aerodynamic force resists the fin opening. Compared to the Kayser design the present fin geometry tested has a negative cant angle for a greater range of fin opening angles.

In the current analysis, the effect of projectile yaw is included in the effective cant angle. Figure 6 shows the effective cant angle as a function of fin opening angle for 5 degrees of yaw. For reference, the effective cant angle for zero yaw is also shown. Since the effect of yaw depends on the orientation of the pitch plane relative to the fins, four different fin orientations relative to the pitch-plane are shown. These four orientations are shown schematically in Fig. 7. Fins A and C are located on the lee and wind sides of the body, respectively. As noted previously, a negative cant angle results in an aerodynamic force that tends to open the fin. In the fully closed position, the effective cant angle of Fin A (lee side) is essentially the sum of the boattail angle and the yaw angles $(7+5=12)$ degrees, while the effective cant angle of Fin $\mathrm{C}$ (wind side) is the difference between the boattail angle and the yaw angles $(7-5=2)$ degrees. Fins B and D have the same cant angle as for the zero yaw case when fully closed. Although the fin cant angle produces a favorable aerodynamic force on the lee side when in the fin is in the closed position, the fin cant angle is positive when the fin is fully deployed. Smooth attached flow (both windward and leeward) is assumed for the calculations as the application of three dimensional flow models very quickly complicates the analysis. This assumption is also more reasonable for small projectile angles of attack, which for this configuration might be considered less than 5 degrees. Projectile yaw levels observed were 2.5 to 3 degrees. Since projectile yaws were not continuously monitored throughout the flight, it's possible that 5 degree levels may have occurred. Maximum projectile 


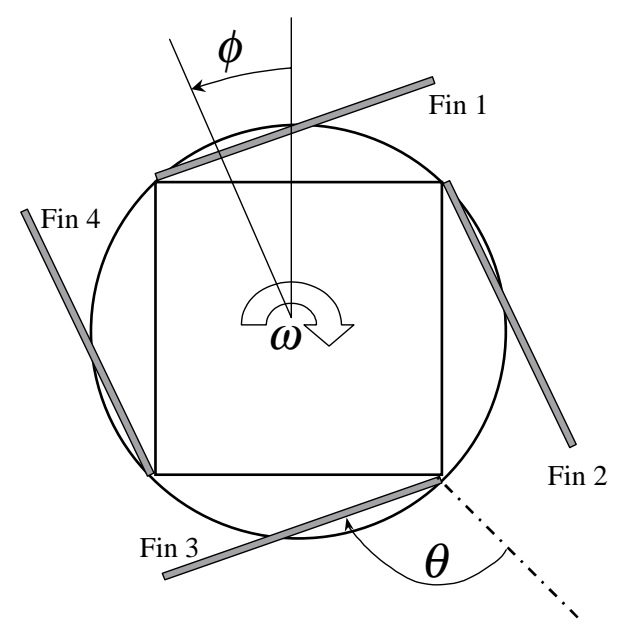

Fig. 3. Schematic of fin opening.
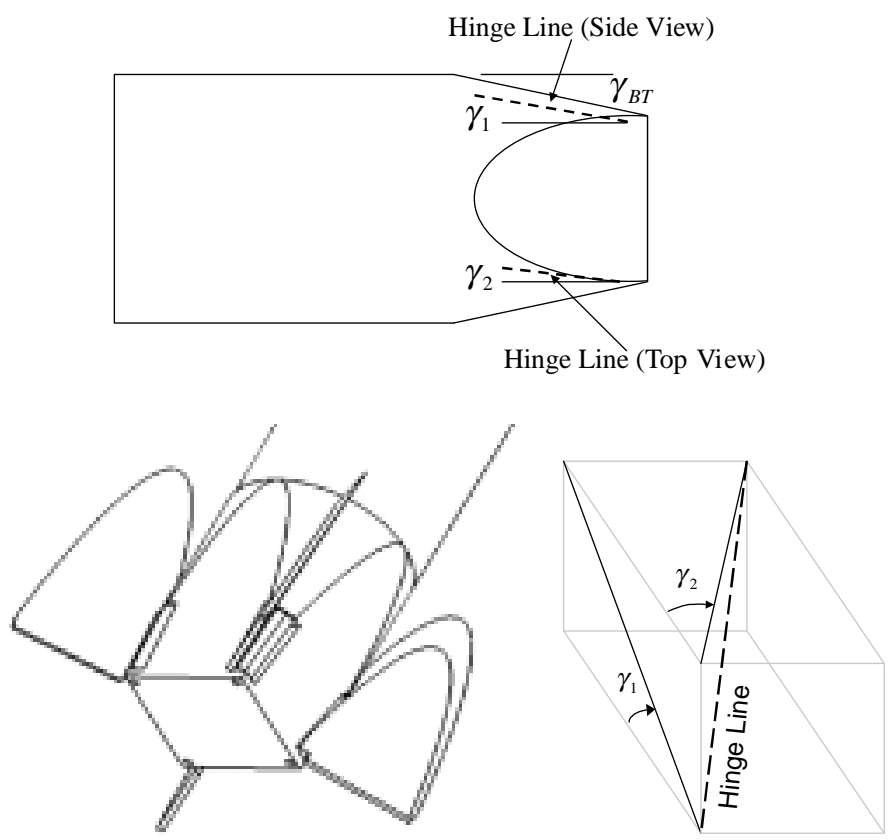

Fig. 4. Views of the compound angle of the hinge line.

yaws are normally seen at the trajectory distances the cameras covered.

Fin D has a positive fin cant angle through nearly the last 90 degrees of the fin opening angle, which Fin B has a negative fin cant angle for all fin opening angles. It is interesting to note that the average cant angle for all four fins is equal to the zero yaw cant angle for all fin opening angles.

The fin blades for the Kayser design typically do not have a symmetrical cross section since the part of the exposed surface when the fins are fully closed is cut to conform to the cylindrical geometry of the projectile. This results in a leading edge bevel on the fin that produces an aerodynamic force that opposes the opening of the fin. This aerodynamic effect is also included in the present aerodynamic model. With this addition to the model, the aerodynamic force can be written in the following form:

$$
F_{\text {aero }}=F_{\delta} \delta_{r}+F_{\text {bevel }}
$$

The leading edge bevel is effectively oriented at the boattail angle of the projectile. Using the wedge pres- 


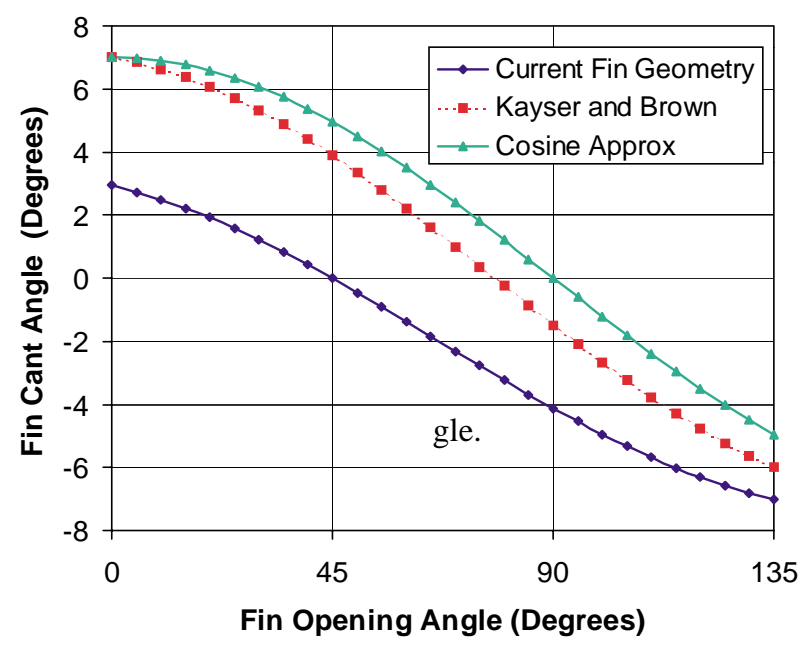

Fig. 5. Effective fin cant angle as a function of fin opening angle.

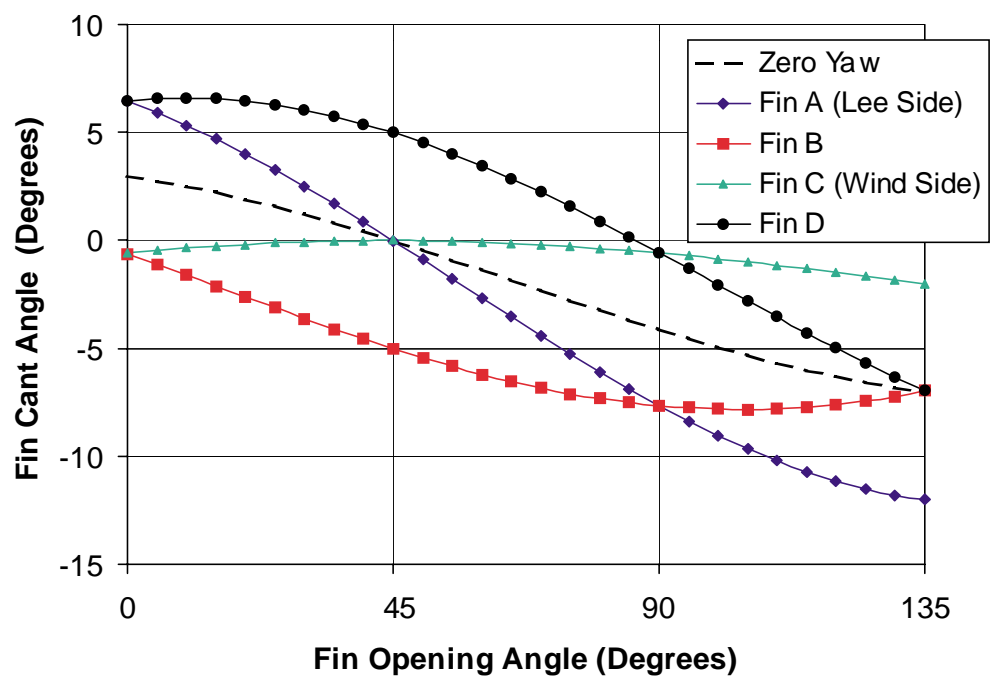

Fig. 6. Effective fin cant angle as a function of fin opening angle, 0 and 5 degrees yaw and zero spin.

sure from compressible flow theory and computing the bevel area, the aerodynamic force due to the bevel can be determined.

The fin force due to fin cant was determined by using simple two-dimensional compressible flow theory for the lift of a flat-plate [2].

$$
F_{\delta}=\frac{8 \rho_{\infty} V_{\infty}^{2} A_{f i n}}{\sqrt{M_{\infty}^{2}-1}}
$$

This approach yields values of the fin force that are similar to those used by Kayser and Brown in their analysis. For the current configuration, $F_{\delta}=9985 \mathrm{~N} / \mathrm{rad}$ and $F_{\text {bevel }}=193 \mathrm{~N}$.

\section{Results and discussion}

Two 34-kg composite projectiles with Kayser fins were fabricated and fired at the Transonic Experimental Facility of Aberdeen Proving Ground. These projectiles used an aluminum boattail portion coupled with a composite body and ogive. They also carried a payload that matched the payload mass found on the M483. The purpose of these firings was twofold. The first was to verify that the composite body was structurally sound for M119A2 (zone 7 Red - designated 7R) charge loads and their corresponding accelerations. The second was to assure that the fins would deploy and the round would fly as expected over the short trajectory of the range. 


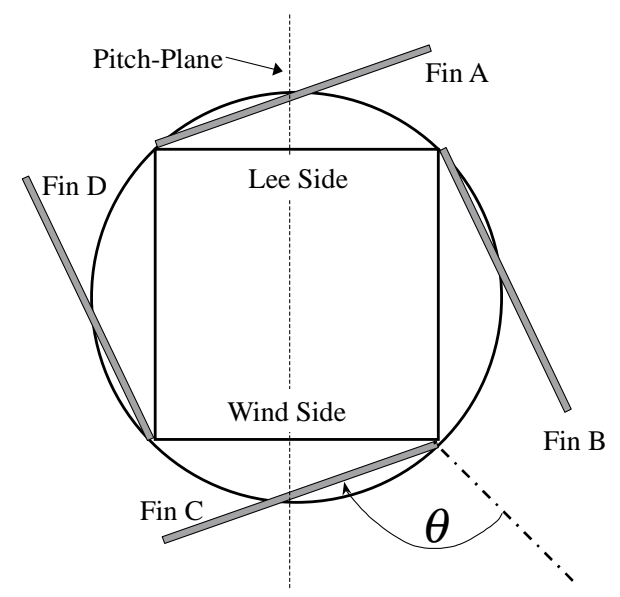

Fig. 7. Schematic showing orientation of Fins A through D relative to pitch-plane.

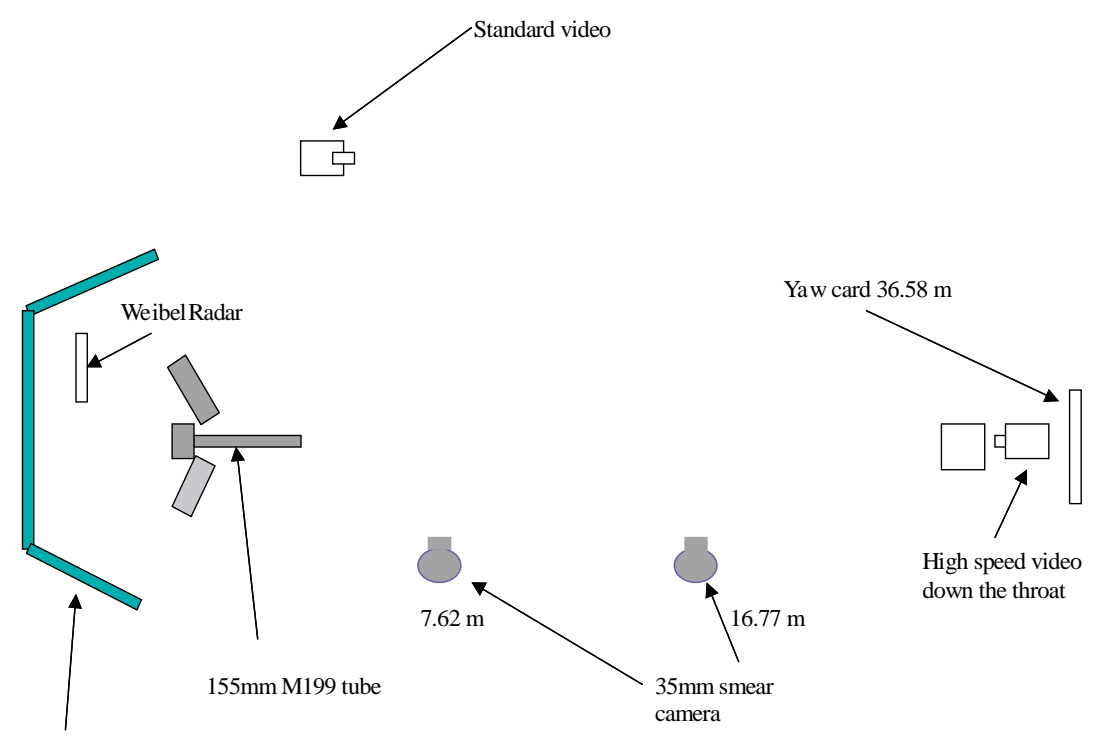

Firing barricade

Fig. 8. Schematic showing orientation of test setup.

The projectile firings used the following instrumentation: 2 cameras, a high speed digital camera, and a yaw card, as well as pressure gages and wide angle video coverage. A Weibel radar was used to monitor the velocity. The zone 7R charge produced a muzzle velocity of $740 \mathrm{~m} / \mathrm{s}$ for the $34 \mathrm{~kg}$ shell. The projectiles were fired from an M199 cannon. A sketch of the test setup is shown in Fig. 8.

The firings produced mixed results. The composite bodies demonstrated that they are structurally adequate. The fin opening behavior produced less satisfactory results. Reviewing the film and yaw card data, it appeared that only one of the 4 fins both deployed and locked.

Figure 9 is a smear photograph at $16.77 \mathrm{~m}$ and shows what appears to be 2 fins almost fully open. The fins look undamaged, though their angular position is difficult to determine simply from a normal view. The yaw angle measured is approximately 5 degrees. The video looking down the gun tube offers the best information as to how the fin is behaving. Frames taken from the video and shown in Fig. 10 illustrate the oscillation of the fin. To increase visibility, the photos have white lines denoting the fin state. Unfortunately there are no trajectory location markers to tie the images. The upper left frame shows the projectile nearest the muzzle and 


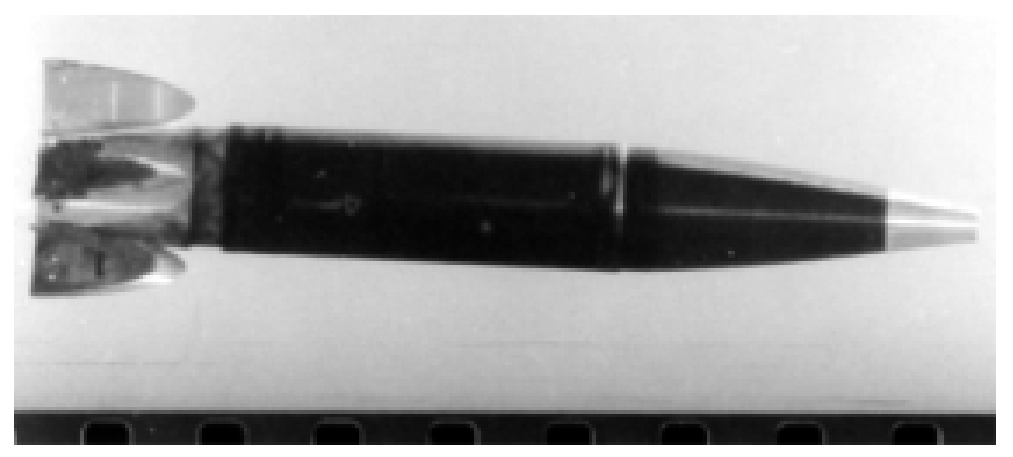

Fig. 9. 34-kg projectile at $16.77 \mathrm{~m}$ from the muzzle.
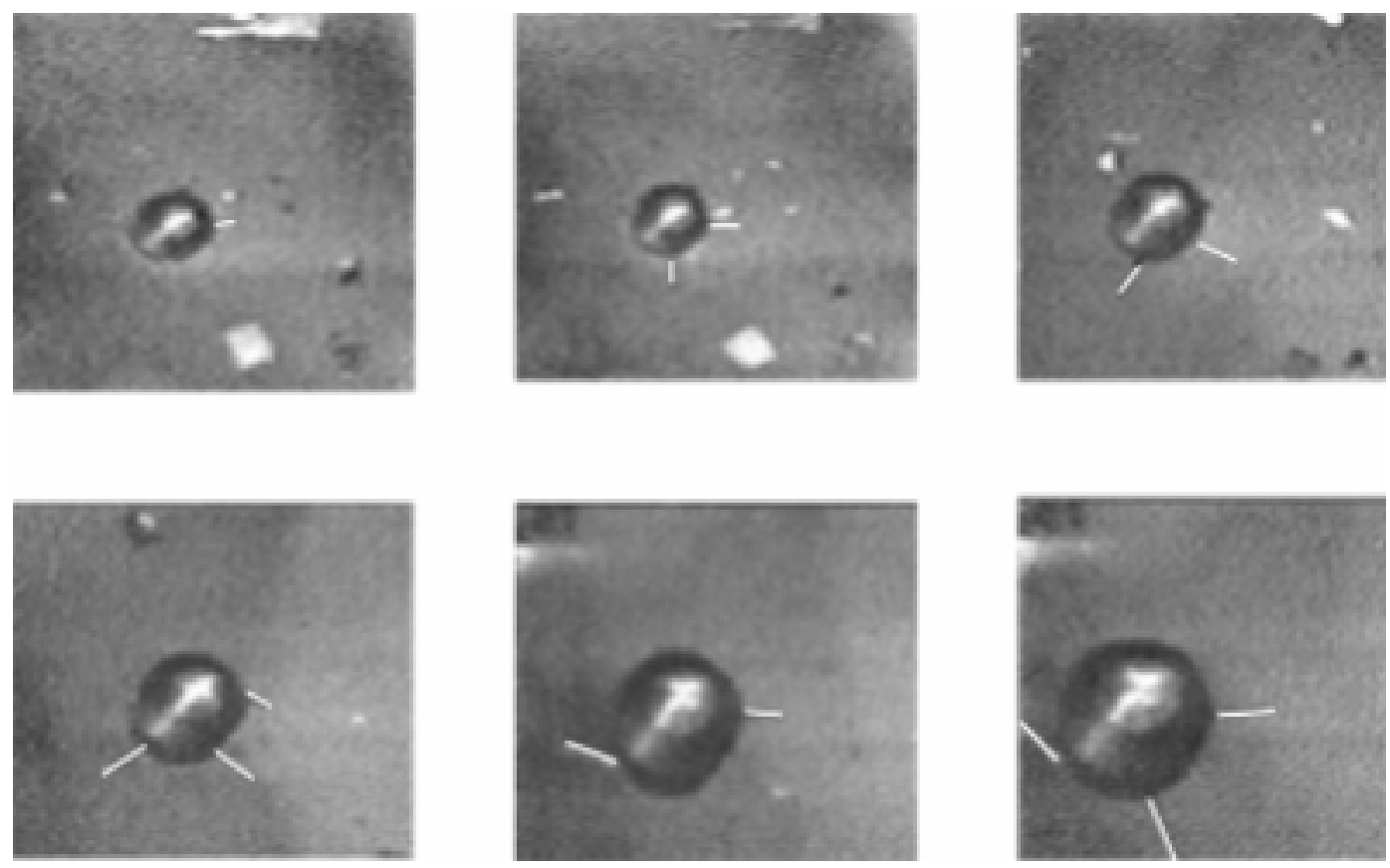

Fig. 10. Fin opening motion over a $36.5 \mathrm{~m}$ trajectory.

the lower right shows the fin state nearest the camera (roughly $36.5 \mathrm{~m}$ from the muzzle). The frames lack sufficient resolution to make accurate reliable angular measurements of the fins angles relative to the projectile body. The fin opening model, developed from the previous equations, was used to interpret the firings. Figure 11 shows the results from the application of this model for a projectile spin rate of $15.6 \mathrm{~Hz}$ and zero yaw. For the conditions examined here, the model predicts that the fin blades open to their fully deployed position within $10 \mathrm{~ms}$. This corresponds to about $7.5 \mathrm{~m}$ of flight or 60 degrees of rotation of the projectile. The fin opening angle is given as the angular difference between the fully deployed position and the instantaneous angle of the fin (as shown in Fig. 2.)
Review of the smear photographs show that the projectile was yawed shortly after launch. The model was applied to determine whether the yaw retarded some fins from opening. The analysis assumes constant yaw amplitude over the period of interest (about $40 \mathrm{~ms}$ ), but the projectile rotates relative to the pitch-plane at $15.6 \mathrm{~Hz}$ (the measured spin rate). Figure 12 shows the results of the analysis for a yaw angle of 5 degrees. Initially, Fin 1 is located on the lee side of the body and Fin 3 is located on the windward side of the body. Fins 2 and 4 are oriented initially 90 degrees from the pitch-plane, such that Fin 2 will be located on the wind side of the body and Fin 4 will be located on the lee side of the body after the projectile initiates 90 degrees of roll due to its spin rate as seen in Fig. 3. 


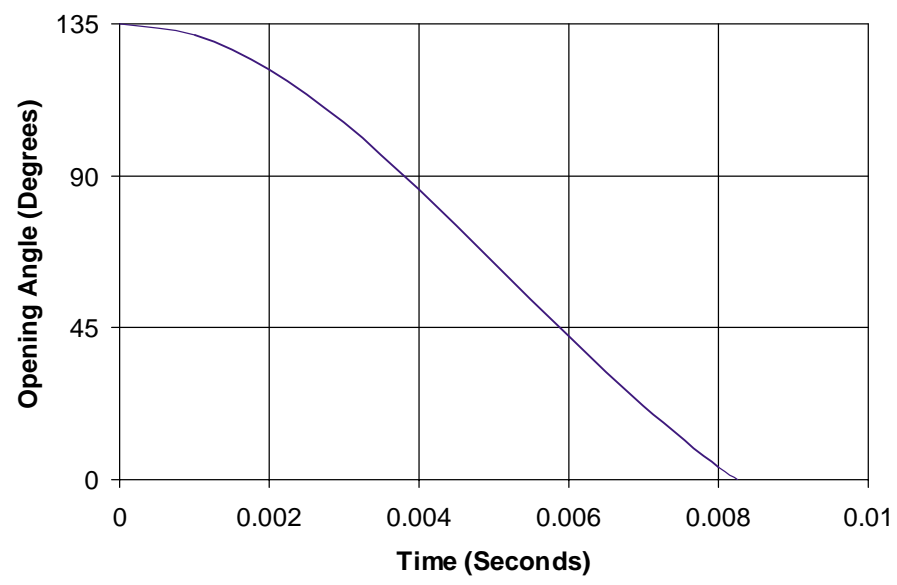

Fig. 11. Fin opening angle as a function of time, zero-yaw, $15.6 \mathrm{~Hz}$ spin rate.

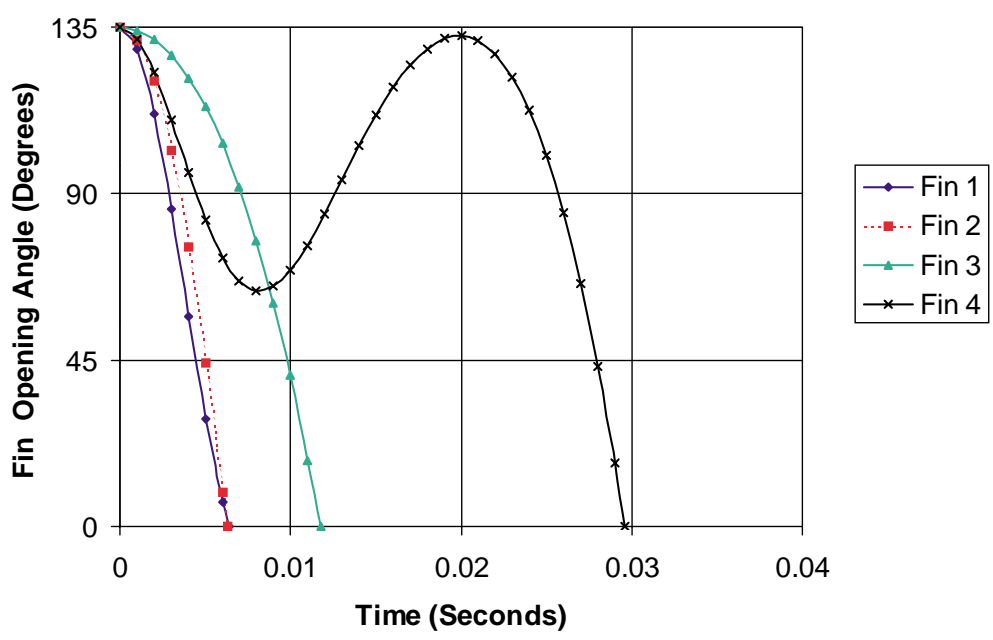

Fig. 12. Fin opening angle as a function of time, five degrees of yaw, $15.6 \mathrm{~Hz}$ spin rate.

The model results indicate that Fins 1 and 2 deploy rapidly and Fin 3 is slightly delayed. The opening of Fin 4 is significantly longer than the other three fins. Only as Fin 4 rotates through the leeward plane does the fin fully deploy. Despite the slow opening of Fin 4, the model shows that the all the fins fully deploy after the body spins through a half-rotation. These results demonstrate that the free-stream angle of attack is an important factor when considering fin deployment.

The presence of yaw can either accelerate or retard the opening of the fin blades. In this case, Fins 1 and 2 open more quickly due to the presence of yaw, because the effective cant angle of the fin is increased by the yaw. For Fin 1, which is initially located on the lee side of the body, the initial cant angle of the fin is nearly 12 degrees, since the yaw adds an additional 5 degrees to the existing cant angle of the fin. However, for Fin 3 , the initial effective cant angle of the fin is reduced to only 2 degrees due to the yaw. Fin 4 shows the most significant effect of yaw. In this case, the yaw initially has no effect on the effective fin cant angle since the fin blade is aligned with the pitch plane when fully closed. As the fin begins to open due to the centrifugal force, the effective fin cant angle quickly becomes positive and the fin opening is retarded. Complete opening of Fin 4 is not possible until the projectile rotates 90 degrees and Fin 4 is on the lee side of the body.

The deployment of the fins is also accelerated by increasing the spin rate of the projectile. Figure 13 shows the fin opening angle as a function of time for a projectile spin rate of $30 \mathrm{~Hz}$. For this increased spin rate, the fin opening angle shows a monotonic decrease in the fin opening angle until the fin is fully deployed. The effect of increasing the spin rate is two-fold. First, the larger 


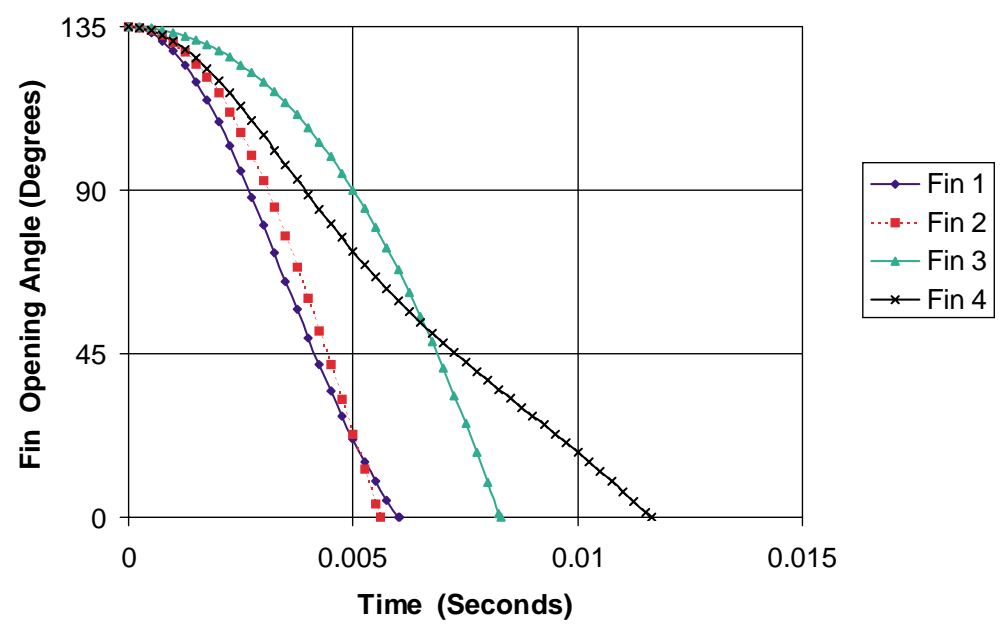

Fig. 13. Fin opening angle as a function of time, five degrees of yaw, $30 \mathrm{~Hz}$ spin rate.

spin rate increases the centrifugal force that accelerates the fin opening. A secondary effect of the increased spin rate is that the fins are rotating more quickly with respect to the pitch-plane. Fins 3 and 4, whose deployment is delayed due to the yaw, rotate more quickly into a position where the aerodynamic forces due to the yaw have a more beneficial effect. Though the actual time for deployment of Fin 4 is decreased for a spin rate of $30 \mathrm{~Hz}$, it still takes approximately one third of a rotation to deploy the fin as compared to nearly one half of a rotation for a spin rate of $15.6 \mathrm{~Hz}$.

The level of difficulty in increasing the projectile spin rate is uncertain. The slip-band obturator used takes into account the melting temperature of the obturator and assumes a frictional coefficient between the obturator band and the polyethylene band seat. Changes such as removing the polyethylene and increasing the projectile surface roughness in the obturator slot should increase the starting torque and the resultant spin. But these solutions are unproven and they bear research and validation.

One explanation for the failure of the fins to fully deploy and lock is that the fin detent pins were not adequate to lock or lock securely enough to hold the fins fully open. This explanation does not account for the fact that the images of the fin larger opening angles (greater than 1.9 radians) were infrequent in a film review. A review of the down-bore photos should show opening angles greater than 1.9 radians for most of the fins over the course of the trajectory monitored. The nonlocking or nonsecuring detent pin explanation is somewhat consistent with the fin motions that were observed. Three of the four fins can be seen closing from semi-open position. And of course one fin for each firing was verified deployed and locked at 135 degrees. Whether the 3 semi-open fins ever reached the fully open state remains unanswered. The round has not completed a full revolution along its trajectory by the last camera location, not all the fins have been exposed to the leeward flow. This flow is conducive to fin opening.

Though the analysis shows the retarded opening of one of the fins, the analysis does not appear to fully corroborate the fin behavior observed in the video. The fin opening model with yaw was applied to examine the behavior of the fins if no locking mechanism were present. Two conditions for the fin were considered that represented extremes: 1) The fin has a perfectly elastic collision with the fin stop or, 2) the fin collision is totally inelastic and the fin comes to a complete slow stop (while not locking) at the fin stop. Figure 14 shows the two oscillatory behaviors. It is difficult to imagine that the fin would come to a stop and the locking pins would not engage (situation 2), but it is possible these pins are damaged by the high pressure chamber environment.

As mentioned previously, the video indicated that the projectile was yawed during the fin opening event. Figure 15 shows the response of each of the four fins in the presence of 5 degrees of yaw when no locking mechanism is present. Early in the event, prior to the fin reaching the fully opened position, the fin motion is identical to the locking case (Fig. 6). After reaching the fully opened position, the fins oscillate between the fully open and partially opened positions. After approximately one projectile body rotation, the motion becomes periodic and a phase shift is predicted for the fin motions. In contrast to the zero yaw case, the presence of yaw enhances the fins' opportunity to reach 


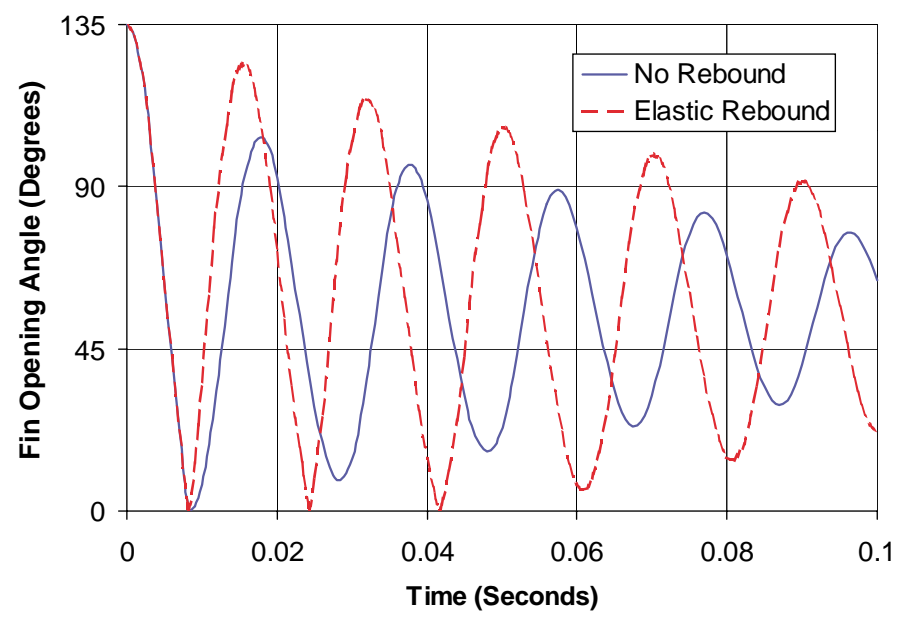

Fig. 14. Fin opening angle as a function of time, no locking mechanism, zero yaw, $15.6 \mathrm{~Hz}$ spin rate.

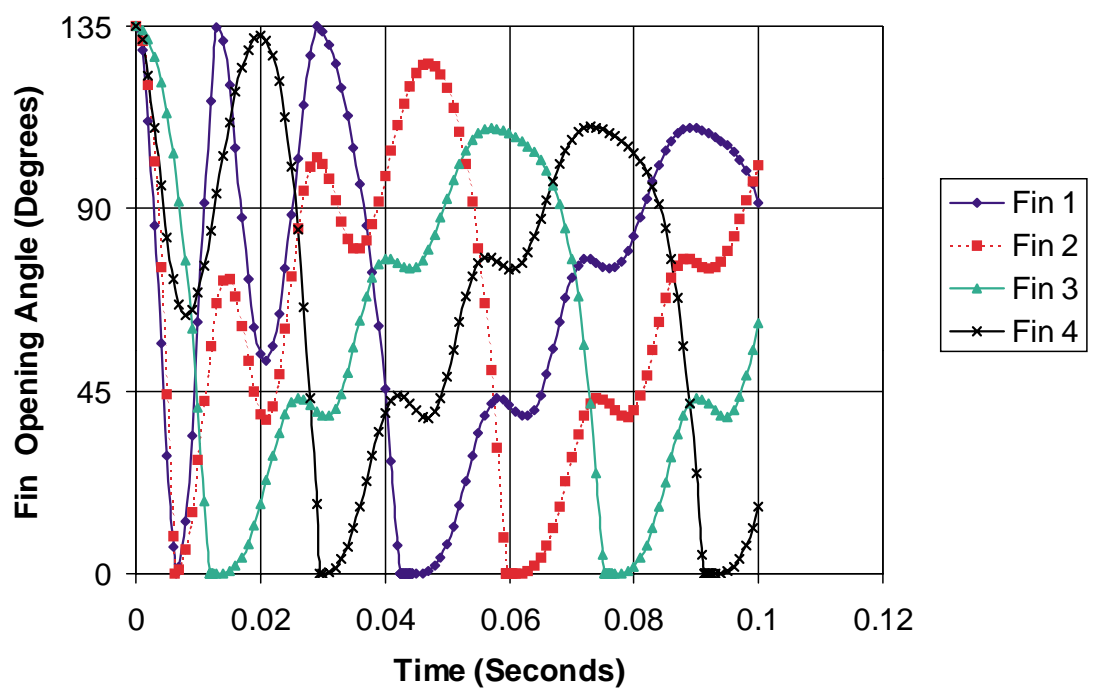

Fig. 15. Fin opening angle as a function of time, no locking mechanism, 5 degrees of yaw, $15.6 \mathrm{~Hz}$ spin rate.

their fully open and locked position once during each rotation of the projectile.

The results in Fig. 15 were obtained assuming an inelastic rebound of the fin against the stop. When an elastic rebound is considered, the motion of the fin is somewhat more complicated than the inelastic rebound. Figure 16 shows the motion of Fin 2 for both elastic and inelastic rebound. Although the oscillations of the fin are larger for the elastic rebound case, the general behavior of the fin is similar for both cases. In particular, the fin will only reach the fully deployed position when the fin is close to the leeward side. For the inelastic rebound case, the fin reaches the fully deployed position only once during each rotation of the projectile. For the elastic rebound case, there are two to three instances where the fin reaches the fully deployed position for each rotation of the projectile.

\section{Conclusions}

The analysis of the fin opening event has been used to provide some interpretation of the results of the experimental firings. In the absence of yaw, the analysis indicates that the fins should open and lock soon after launch. The presence of yaw can slightly delay the opening of individual fins. However, as the projectile rotates relative to the pitch-plane, the effect of yaw will eventually provide sufficient aerodynamic force to cause all of the fins to fully deploy within a projectile 


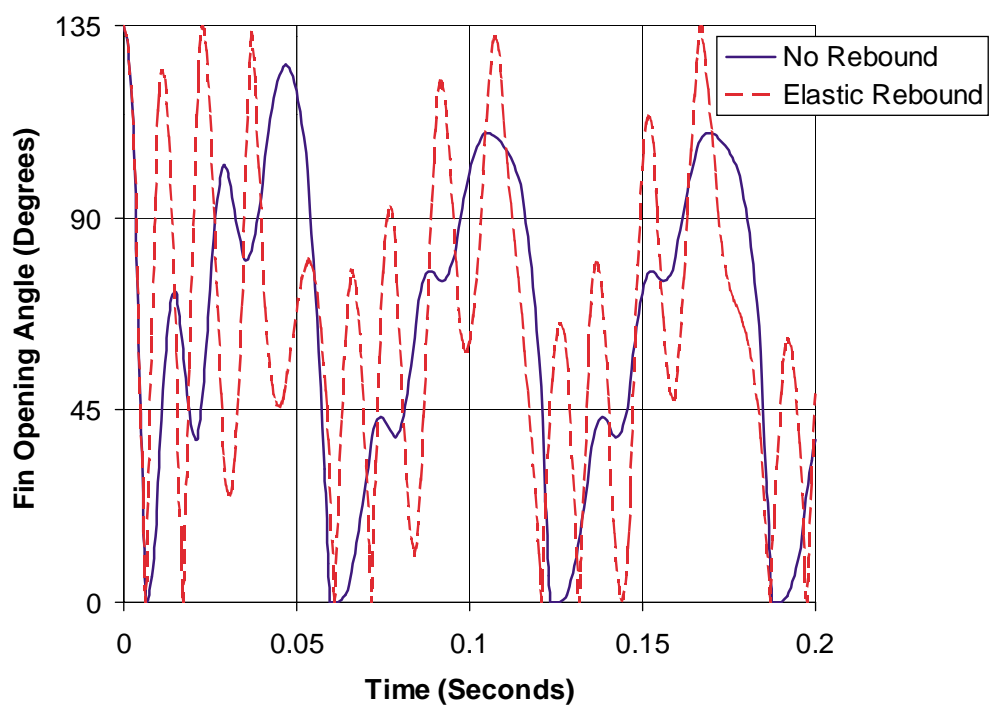

Fig. 16. Fin angle response to Impact with Fin Stop as a function of time, no locking mechanism, 5 degrees of yaw, $15.6 \mathrm{~Hz}$ spin rate.

rotation. Since the observed motion from the video of the experimental test indicates that the fins open but do not lock, it appears that the locking mechanism either failed or did not engage for at least some of the fins. Oscillations of the fins between the fully deployed and partially open positions are possible when the fins do not lock.

Based on the modeling results as well as review of the range film, and engineering judgment, some modifications have been incorporated for future firings. Locking pins with an increased spring tension have been selected and are scheduled for use. Additionally, a higher projectile spin rate, perhaps near $30 \mathrm{~Hz}$, is also planned. An increased firing elevation has also been suggested to ensure that each of the fins will have an increased opportunity to rotate through the leeward flow environment, which is conducive to fin opening.

\section{Acknowledgements}

The origination of this design is an adaption of a fin design by Lyle Kayser. It is an extension of the principles developed under the HICAP program. James Bender, the 75-lb shell program originator, sponsored the tests, gave guidance and assisted in the analysis. The ARL machine shop, specifically, Gary Sprenkle, did a masterful job in fabricating the projectiles and offering constructive suggestions. And lastly, the personnel at Transonic Experimental Facility, were able to test the design in a timely manner, and their efforts are appreciated.

\section{References}

[1] E. Dickinson, The Effect of Boattailing on the Drag Coefficient of Cone Cylinder Projectiles At Supersonic Velocities, Ballistic Research Laboratory Memorandum Report 842, Aberdeen Proving Ground, MD, November, 1954.

[2] John, E.A. James, Gas Dynamics, Allyn and Bacon Inc., Boston, MA, 1969.

[3] L. Kayser and T. Brown, Fin Motion After Projectile Exit From Gun Tube, U.S. Army Ballistics Research Laboratory Report, BRL-TR-3375, Aberdeen Proving Ground, MD 21005, July, 1992. 

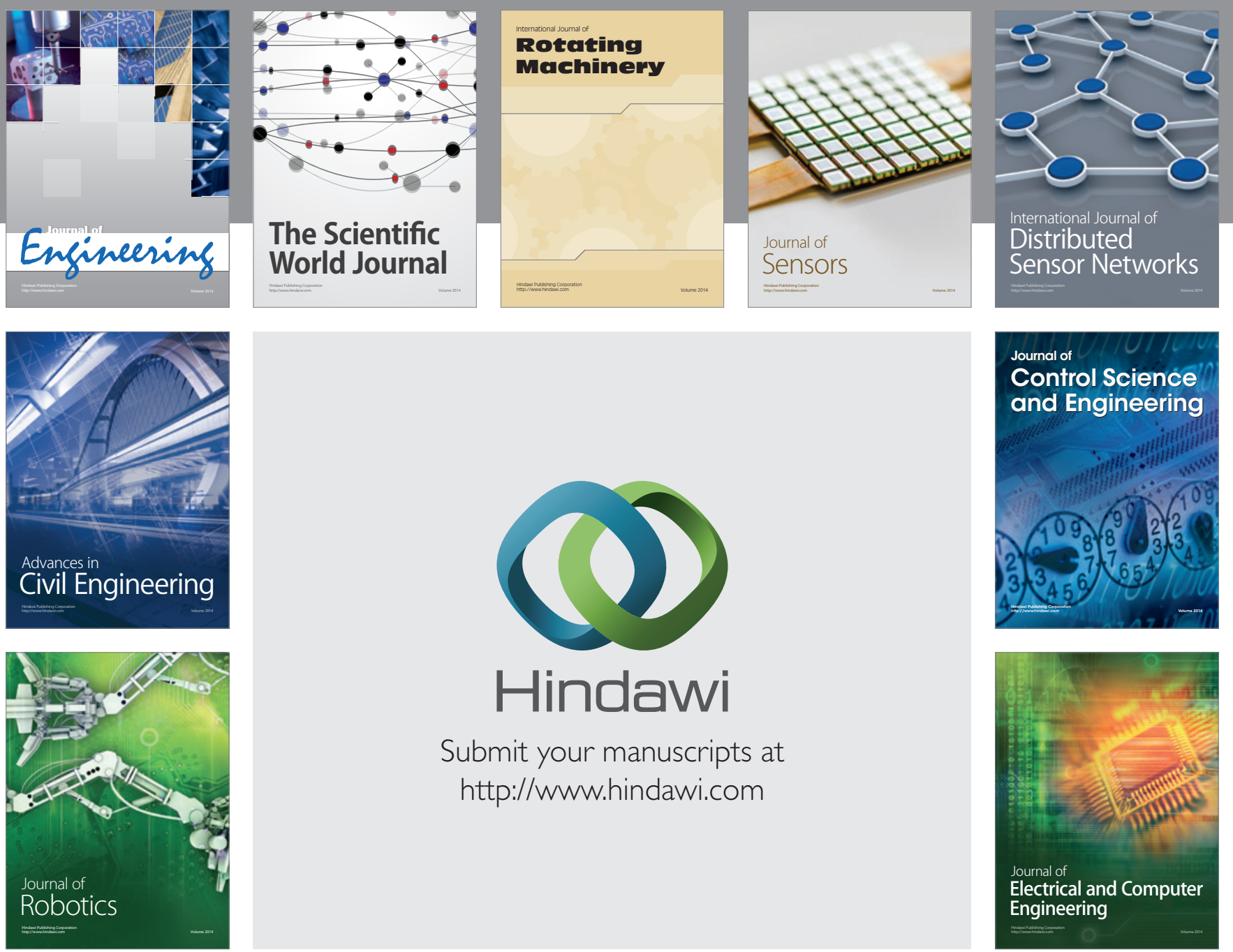

Submit your manuscripts at

http://www.hindawi.com
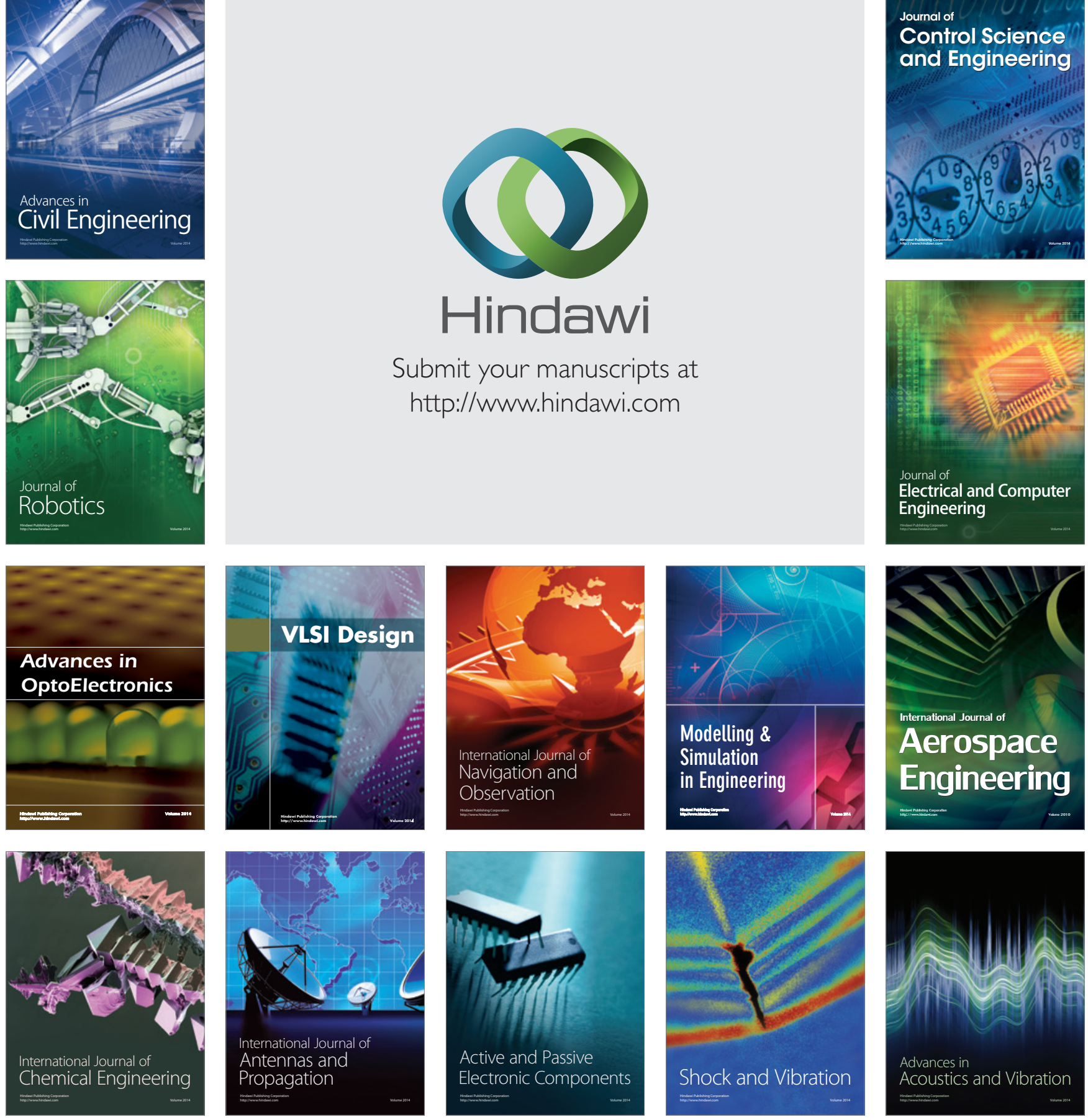